\title{
Survivin-2B promotes autophagy by accumulating IKK alpha in the nucleus of selenite-treated NB4 cells
}

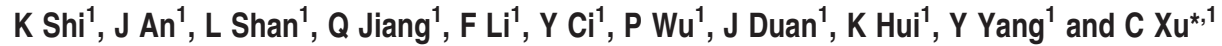

Survivin-2B, a known splice variant of survivin, has been reported to promote cell death in some cancer cells, although it keeps prosurvival function in others, and the mechanisms are unclear. In this report, we discovered that selenite, an antitumor agent, switched protective autophagy to apoptosis in NB4 cells. In this process, the level of survivin-2B was decreased and the interaction between IKK alpha and survivin-2B in the nucleus was attenuated, which further led to the decrease of nuclear IKK alpha. As a result, P73, a known transcript factor of UVRAG, was downregulated. Therefore, the expression of UVRAG, one of the initiators of autophagy, was inhibited. The regulatory status of survivin-2B was also proved in NB4 cells after different chemicals' exposure and in other tumor cell lines (Jurkat, HCT116). Finally, experiments in vivo confirmed that the alterations of survivin-2B, IKK alpha, P73 and UVRAG were the same as that in vitro. Taken together, survivin-2B promoted autophagy and further regulated cell death by accumulating and stabilizing IKK alpha in the nucleus.

Cell Death and Disease (2014) 5, e1071; doi:10.1038/cddis.2014.34; published online 20 February 2014

Subject Category: Cancer

Autophagy is the sequestration of a cell's organelles and proteins into autophagosomes for degradation in lysosomes; it has an important role in regulating tumorigenesis. Some tumor cells are killed by autophagy, whereas autophagy works as a 'bodyguard' to prevent the death of others. ${ }^{1}$ Therefore, investigations into the mechanisms by which autophagy determines the fate of tumor cells could be important for tumor prevention and therapy.

Selenium is an essential trace element in humans. Emerging evidence has demonstrated that a super-nutritional intake of selenite can inhibit tumor cell growth. Many studies focusing on the effects of selenite on tumor xenograft in animal models have also shown that selenite possesses some antitumor activity. ${ }^{2-8}$ Thus, a clarification of the mechanisms by which selenite induces tumor cell death is needed. Our previous study showed that the addition of selenite switched cells from undergoing protective autophagy to apoptosis, and the mechanism was through the downregulation of Hsp90. ${ }^{9}$ In addition, reports using other cell lines have shown that selenite promotes both autophagy and apoptosis. ${ }^{10-13}$ On the basis of these previous studies, elucidating the relationship between autophagy and apoptosis in cancer cells after selenite exposure is necessary.

Survivin is a member of the inhibitors of apoptosis (IAP) family. In recent years, more and more reports have demonstrated that survivin participates in the regulation of the autophagy network. ${ }^{14-17}$ Splicing variants of survivin have been identified and, among them, survivin delta $X 3$ regulated apoptosis similarly to wild-type survivin, whereas survivin-2B demonstrated a proapoptotic role in some cancer cells. ${ }^{18,19}$
Studies have shown that AML patients have a lower survival rate if higher levels of survivin-2B are observed, ${ }^{20}$ whereas in EU-3 cells, an ALL cell line, survivin-2B can have a proapoptotic role. ${ }^{21}$ The current study found that selenite downregulated the expression of survivin-2B and switched protective autophagy to apoptosis in NB4 cells. Our investigation thus revealed the mechanisms by which survivin-2B regulated autophagy and apoptosis in selenite-treated NB4 cells.

\section{Results}

Selenite inhibited protective autophagy and promoted apoptosis. After cells were exposed to $20 \mu \mathrm{M}$ selenite for various lengths of time, western blotting was performed to detect markers of apoptosis and autophagy. Cleavages of caspase 3 and PARP were observed in NB4 cells, whereas the increase of P62 and the decrease of Beclin-1 and LC3II reflected that selenite exposure inhibited autophagy in NB4 cells (Figure 1a). Meanwhile, observation by electron microscopy confirmed that the number of double-membrane vesicles known as autophagosomes was decreased (Figure 1b), whereas cells that were indirectly labeled with anti-LC3 antibodies and FITC-conjugated anti-mouse secondary antibodies exhibited punctate aggregation and remained disperse after selenite exposure (Figure 1c). Additional experiments demonstrated that inhibition of autophagy with 3-methyladenine or bafilomycin A1 could enhance selenite-induced apoptosis (Figures 1d, $\mathrm{g}$ and $\mathrm{h}$; Supplementary Figures S1A and B). Meanwhile, we also

\footnotetext{
${ }^{1}$ State Key Laboratory of Medical Molecular Biology, Institute of Basic Medical Sciences and School of Basic Medicine, Department of Biochemistry and Molecular Biology, Peking Union Medical College and Chinese Academy of Medical Sciences, Beijing, China

${ }^{*}$ Corresponding author: C Xu, Biochemistry and Molecular Biology, National Laboratory of Medical Molecular Biology, Institute of Basic Medical Sciences, CAMS \& PUMC, Dong Dan San Tiao wu hao, Beijing 100005, China. Tel: +86 1069 156445; Fax: +86 1069 156445; E-mail: cmxu@ibms.pumc.edu.cn

Keywords: survivin-2B; selenite; apoptosis; autophagy

Abbreviations: $\mathrm{As}_{2} \mathrm{O}_{3}$, arsenic (III) oxide; $\mathrm{PI}$, propidium iodide; YM155, a novel suppressant of survivin; 3MA, 3-methyladenine; $\mathrm{HE}$, hematoxylin-eosin; $\mathrm{H}_{2} \mathrm{O}_{2}$, hydrogen peroxide

Received 12.9.13; revised 12.1.14; accepted 15.1.14; Edited by GM Fimia
} 

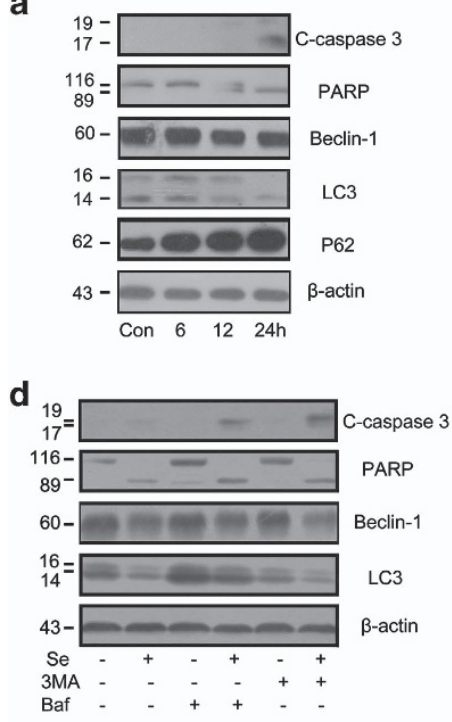

b

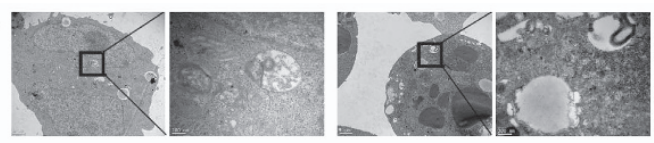

C

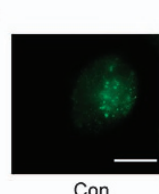
Con

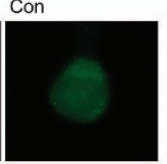

$\mathrm{Se}$

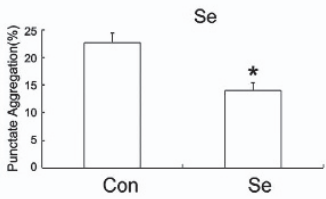

e

f
Con

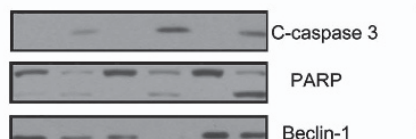

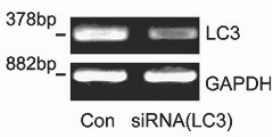

$\mathrm{Se}$ SiRNA(LC3) SiRNA(Bec)
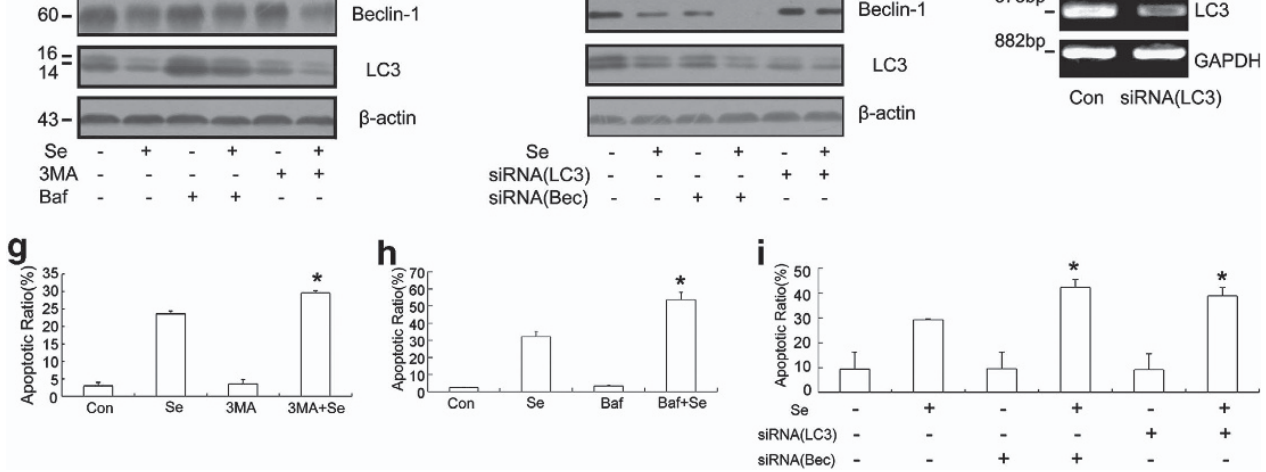

Figure 1 Selenite switched protective autophagy to apoptosis. (a) Sodium selenite regulated both apoptosis and autophagy in NB4 cells. After the cells were exposed to $20 \mu \mathrm{M}$ selenite for the indicated period, whole-cell lysates were extracted, and apoptosis and autophagy markers were detected by western blotting. (b) Electron microscopy reflected alteration of autophagy in $20 \mu \mathrm{M}$ selenite-treated NB4 cells. (c) Selenite inhibited LC3 punctate aggregation in NB4 cells. After cells were treated with selenite for $24 \mathrm{~h}$, LC3 was indirectly labeled with antibodies. The images were visualized with a Zeiss microscope. The degree of punctate aggregation was represented as LC3 dotpositive cells (with more than 10 dots) $/ 100$ cells. The value was represented as mean \pm S.E.M. $(n=3)$. The scale bar represents $10 \mu$ m. ${ }^{*} P<0.05$. (d) Inhibition of autophagy also enhanced selenite-induced apoptosis in NB4 cells. NB4 cells were pretreated with 3-methyladenine (3MA) (1 mM) or bafilomycin A1 (10 nM) for $1 \mathrm{~h}$. After selenite treatment for $24 \mathrm{~h}$, the levels of cleaved caspase 3 and PARP were detected by western blotting. (e) Silencing autophagy-related protein obviously enhanced selenite-induced apoptosis. After NB4 cells were transfected with siRNA targeting Beclin-1 or LC3, $20 \mu \mathrm{M}$ selenite was added for another $24 \mathrm{~h}$. The levels of cleaved caspase 3 and PARP were detected by western blotting. (f) RT-PCR assay was carried out for LC3 mRNA level analysis. After siRNA targeting, LC3 was transfected and selenite treated for another $24 \mathrm{~h}$, RNA in NB4 cells was extracted and underwent reverse transcriptional PCR process. Finally, CDNA levels of GAPDH and LC3 were detected by PCR assay. GAPDH was used as a loading control for each treatment. ( $\mathbf{g}-\mathbf{i})$ Inhibition of autophagy resulted in the enhanced apoptosis in NB4 cells. After NB4 cells were transfected with siRNA targeting each sequence or pretreated with $3 \mathrm{MA}(1 \mathrm{mM})$ or bafilomycin $\mathrm{A} 1(10 \mathrm{nM})$ for $1 \mathrm{~h}$, selenite was added and the apoptotic ratio of each treatment was detected by flow cytometry. The value was represented as mean \pm S.E.M. $(n=3) .{ }^{*} P<0.05$

found that autophagic flux was not inhibited by using bafilomycin A1 in NB4 cells. Furthermore, after siRNAs targeting Beclin-1 and LC3 were transfected into NB4 cells and $20 \mu \mathrm{M}$ selenite worked for another $24 \mathrm{~h}$, we discovered that cleavages of caspase 3 and PARP were increased and apoptotic ratio was also promoted (Figures $1 \mathrm{e}$ and i; Supplementary Figure S1C). Results from RT-PCR had confirmed that LC3 transcriptional level was downregulated indeed after siRNA was transfected (Figure 1f). Taken together, selenite inhibited protective autophagy in NB4 cells.

Survivin-2B, IKK alpha and P73 promoted protective autophagy in NB4 cells. To explore the detailed mechanisms preliminarily, we determined the alterations of survivin, survivin-2B, IKK alpha, P73 and UVRAG and discovered that all of them were downregulated (Figure 2a). Meanwhile, we treated NB4 cells with other chemical compounds such as ATRA, $\mathrm{H}_{2} \mathrm{O}_{2}$ and $\mathrm{As}_{2} \mathrm{O}_{3}$ to confirm that the relationship between these proteins and autophagy had universality. $\mathrm{H}_{2} \mathrm{O}_{2}$ apparently downregulated survivin-2B, IKK alpha, P73,
UVRAG, Beclin-1 and LC3II, whereas ATRA and $\mathrm{As}_{2} \mathrm{O}_{3}$ upregulated Beclin-1 and LC3II along with the increase in the activation of survivin-2B/IKK alpha/P73 axis (Figure 2b). As you could see, although survivin decreased after selenite exposure, ATRA could not alter survivin similarly to IKK alpha, P73 and UVRAG. This result helped us explore the potential regulatory status of survivin-2B. Because Beclin-1 and LC3II were necessary for the generation of autophagosomes, all of those findings indicated that the alterations of survivin-2B, IKK alpha and P73 were in conformity with the change in autophagy. To access the direct function of these proteins, we inhibited their expression through siRNA interference and indirectly labeled LC3. After calculating the LC3 dot-positive cells (with more than 10 dots)/100 cells, we discovered that survivin-2B, IKK alpha, P73 and UVRAG could promote autophagy in NB4 cells (Figure 2c). Along with indirect immunofluorescence assay, we extracted proteins after each member of this pathway was knocked down. Results from western blotting also proved that survivin-2B, IKK alpha, P73 and UVRAG had autophagy promotion 

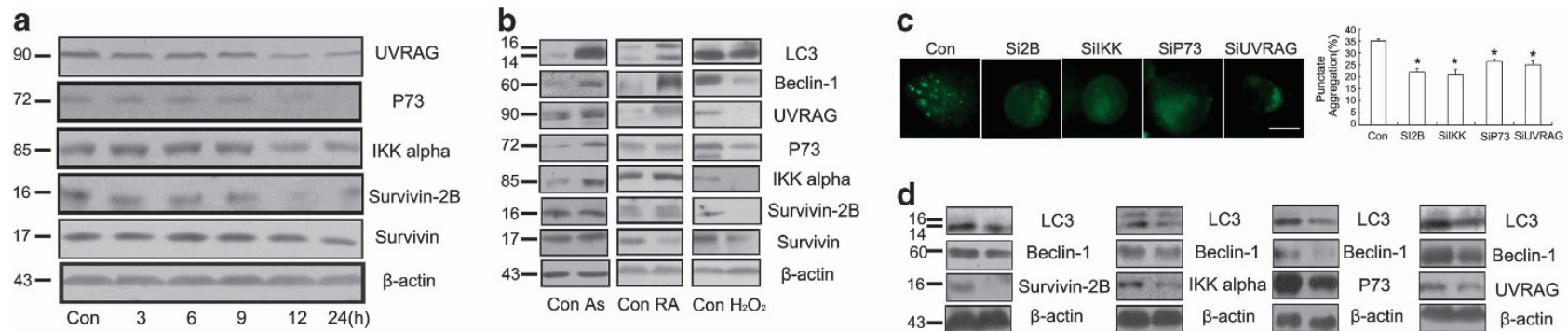

d

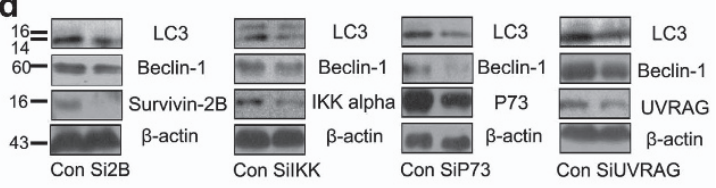

e

f

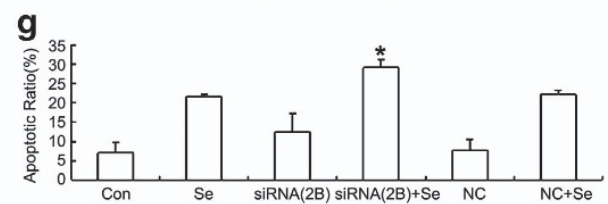

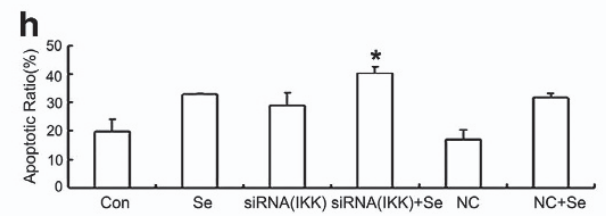

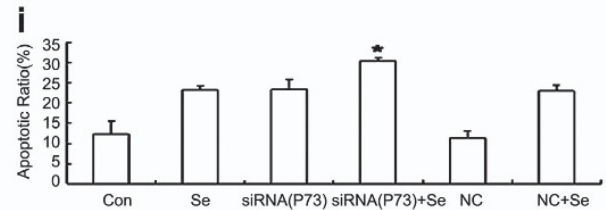

Figure 2 Survivin-2B, IKK alpha and P73 promoted protective autophagy in NB4 cells. (a) Sodium selenite inhibited survivin-2B/IKK alpha axis in NB4 cells. After the cells were exposed to $20 \mu \mathrm{M}$ selenite for the indicated period, whole-cell lysates were extracted, and the levels of survivin-2B, IKK alpha, P73 and UVRAG were detected. (b) $\mathrm{As}_{2} \mathrm{O}_{3}, \mathrm{H}_{2} \mathrm{O}_{2}$ and ATRA altered survivin-2B/IKK alpha axis. After NB4 cells were exposed to $\mathrm{As}_{2} \mathrm{O}_{3}(0.8 \mu \mathrm{M}), \mathrm{H}_{2} \mathrm{O}_{2}(100 \mu \mathrm{M})$ and ATRA $(2 \mu \mathrm{M})$ for $24 \mathrm{~h}$, alterations of survivin-2B, IKK alpha, P73 and UVRAG were detected by western blotting. (c) Survivin-2B, IKK alpha, P73 and UVRAG promoted autophagy. Cells were transfected with siRNA targeting its special sequence; LC3 was indirectly labeled. The images were visualized with a Zeiss microscope. The scale bar represents $10 \mu \mathrm{m}$. The degree of punctate aggregation was represented as LC3 dot-positive cells (with more than 10 dots)/ 100 cells. ${ }^{*} P<0.05$. (d) Survivin-2B IKK alpha, P73 and UVRAG regulated the level of beclin-1 and LC3II. After cells were transfected with special siRNA sequence, selenite worked for another $24 \mathrm{~h}$. Whole-cell lysates were extracted and alterations of beclin-1 and LC3II were detected by western blotting. (e-g) Selenite affected autophagy and apoptosis by regulating the levels of the survivin-2B protein. After NB4 cells were transfected (with siRNA targeting survivin-2B or survivin-2B overexpression plasmids) or pretreated with YM155 $(500 \mathrm{nM})$ for $1 \mathrm{~h}, 20 \mu \mathrm{M}$ selenite was treated for another $24 \mathrm{~h}$ and the apoptotic ratio was detected by flow cytometry. The value was represented as mean \pm S.E.M. $(n=3)$. ${ }^{*} P<0.05$. (h and i) IKK alpha and P73 worked against apoptosis. After being transfected with siRNA targeting either IKK alpha or P73, $20 \mu \mathrm{M}$ selenite worked for another $24 \mathrm{~h}$. Cells were collected and stained with Annexin V/PI for further analysis. The value was represented as mean \pm S.E.M. $(n=3) .{ }^{*} P<0.05$

function (Figure 2d). As we observed that survivin-2B, IKK alpha and P73 were decreased along with the switch from autophagy to apoptosis, we further determined whether these proteins took part in the regulation of apoptosis. YM155, a novel suppressant of survivin, could inhibit survivin and its splice variants' expression. Therefore, Annexin V-FITC/PI staining analysis was used to determine the apoptotic ratio of control and selenite-treated cells that had downregulated, overexpressed or normal expression of survivin-2B. The results indicated that survivin-2B had an antiapoptotic role in NB4 cells (Figures 2e-g; Supplementary Figures S2A-C). Finally, the apoptosis-preventing effects of IKK alpha and P73 were also confirmed through Annexin V/ $\mathrm{PI}$ double staining analysis after silencing either IKK alpha or P73 in NB4 cells (Figures $2 \mathrm{~h}$ and i; Supplementary Figures S2D and E).

Survivin-2B accumulated and stabilized IKK alpha in the nucleus and regulated the switch from autophagy to apoptosis. We had discovered that survivin-2B, IKK alpha and P73 took part in the regulation of protective autophagy. Further experiments were performed to explore the exact mechanisms. GST pull-down assay confirmed that survivin2B could interact with IKK alpha (Figure 3a), whereas immunoprecipitation analysis demonstrated that the interaction of IKK alpha and survivin-2B in NB4 cells was decreased by selenite (Figure $3 b$ ). In addition, we also discovered that the content of IKK alpha in the nucleus was decreased (Figure $3 \mathrm{c}$ ) and the colocalization of survivin-2B and IKK alpha was canceled after selenite treatment (Figure 3d). As we indicated previously, different chemicals could alter the levels of survivin-2B, IKK alpha and P73 along with the changes in autophagy. Therefore, we indirectly labeled IKK alpha and survivin-2B after these chemicals' exposure. Immunofluorescence analysis reflected that the degree of colocalization of IKK alpha and survivin-2B was in line with the extent of autophagy (Figure 3d). To fully demonstrate the autophagy promotion role of survivin-2B, we also analyzed autophagy after overexpression of survivin-2B as we did for apoptosis analysis previously. After calculating the LC3 dot-positive cell ratio, we found that upregulation of survivin-2B expression also prevented selenite inhibitory effects on autophagy in NB4 cells (Figure 3e). Meanwhile, we transfected survivin-2B overexpression plasmids and treated NB4 cells with selenite for $24 \mathrm{~h}$. The results from western blots also proved that survivin-2B prevented selenite inhibitory effects on autophagy (Figure 3f). Furthermore, we combined the use of either survivin transcriptional inhibitor, YM155, or transfecting siRNA targeting survivin-2B and selenite. The results from western blot analysis indicated that 

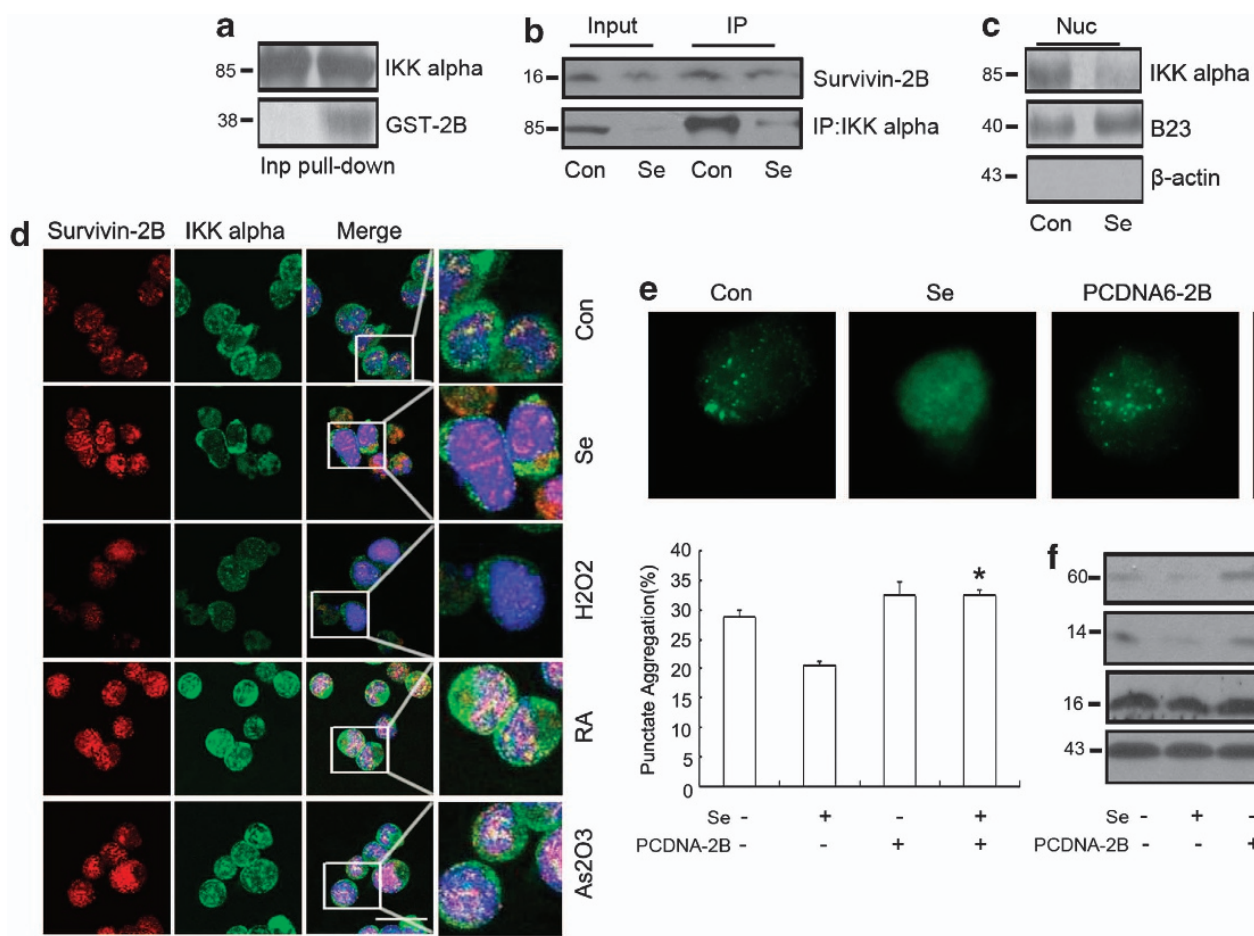

PCDNA6-2B
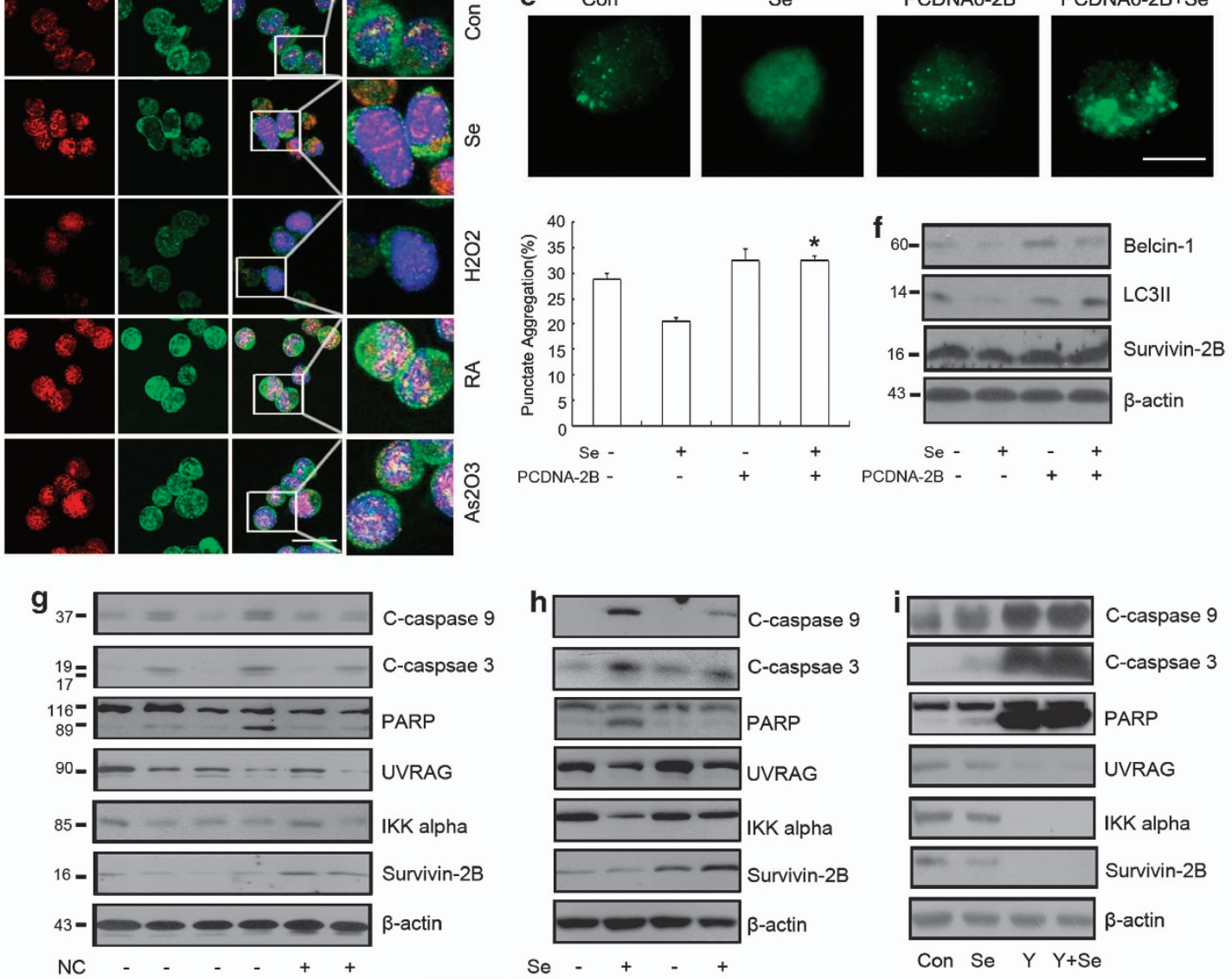
SiRNA

(Survivin-2B)
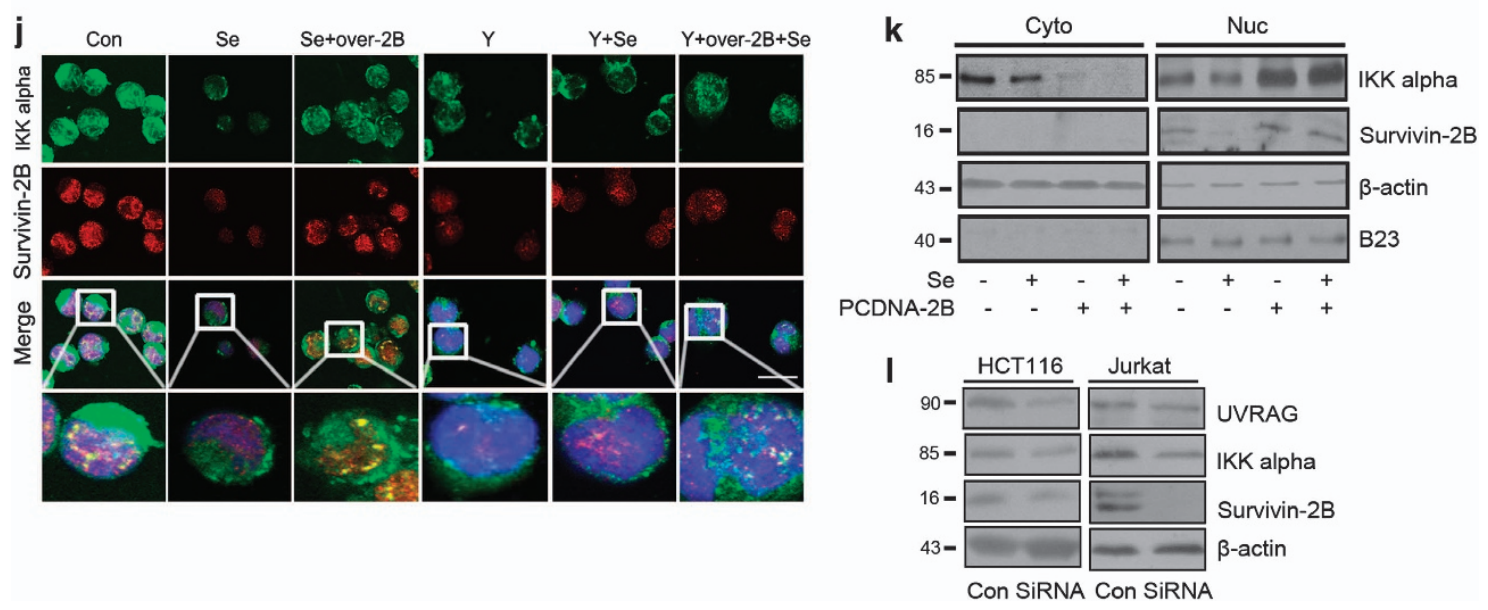
the downregulation of survivin-2B could promote apoptosis degree with enhanced cleavages of caspase 9, caspase 3 and PARP, whereas this combination treatment further inhibited the expression of UVRAG. Inhibition of survivin-2B downregulated the protein level of IKK alpha as well (Figures $3 \mathrm{~g}$ and $\mathrm{i})$. To fully demonstrate the important role of survivin$2 \mathrm{~B}$, survivin-2B overexpression plasmids were transfected. In agreement with our speculation, alterations of IKK alpha, apoptosis- and autophagy-related proteins that were induced by selenite were diminished (Figure $3 \mathrm{~h}$ ). Because we had demonstrated that survivin-2B was mainly localized in the nucleus, we hypothesized that survivin-2B might control the expression of proteins related to autophagy. As there were no reports suggesting that survivin-2B worked as a transcription factor, we inferred that survivin-2B regulated gene expression indirectly. Because we have proved that survivin-2B could interact with IKK alpha and their interaction altered similarly with autophagy, additional experiments were carried out and the results demonstrated that overexpression of survivin-2B could inhibit the decrease of IKK alpha in the nucleus. Moreover, YM155, a known inhibitor of survivin and its family members, further downregulated the content of IKK alpha in the nucleus and on that basis overexpression of survivin-2B rescued the loss of nuclear IKK alpha (Figure 3j). This result also supported that even though survivin might also have a function in cell death determination after selenite exposure, its regulatory status must be independent of survivin-2B. To fully confirm this result, we extracted the cytoplasmic and nuclear fraction of NB4 cells after survivin$2 \mathrm{~B}$ overexpression plasmids were transfected, and found that the increase of survivin-2B finally resulted in the nuclear accumulation of IKK alpha (Figure 3k). Those results suggested that survivin-2B might be necessary for the nuclear localization of IKK alpha. To our knowledge, we are the first to indicate that survivin-2B could regulate IKK alpha. Therefore, several experiments are necessary to prove its universality. Therefore, we transfected siRNA targeting survivin-2B into Jurkat and HCT116 cells and discovered that IKK alpha and UVRAG were also downregulated (Figure 3l).
IKK/P73 participated in the regulation of UVRAG expression. If IKK alpha localization in the nucleus is regulated by survivin-2B, it may be involved in regulating autophagy. Some groups have shown that nuclear IKK alpha is necessary for the stability of P73, a member of the P53 family that directly binds to the promoter of UVRAG and initiates its transcription. As expected, using an immunoprecipitation assay, we demonstrated that P73 interacted with IKK alpha in the nucleus of NB4 cells (Figure 4a). Meanwhile, we indirectly labeled IKK alpha and P73 with primary antibodies and observed obvious colocalization of IKK alpha and P73 in untreated NB4 cells, whereas this colocalization was canceled after selenite exposure (Figure 4b). As expected, the decrease of IKK alpha destabilized P73 and promoted apoptosis with increasing cleavages of caspase 9, caspase 3 and PARP, whereas downregulation of IKK alpha further inhibited autophagy by decreasing Beclin-1 and UVRAG (Figure 4d). Moreover, the CHIP assay explored that P73 could bind to the promoter of UVRAG, and this binding was attenuated after selenite exposure (Figure 4c). Similarly, the decrease of P73 expression could apparently promote selenite-induced apoptosis with enhancing cleavages of caspase 9 , caspase 3 and PARP and further inhibited autophagy through the downregulation of Beclin-1 and UVRAG (Figure 4e).

\section{Survivin-2B/IKK alpha/P73/UVRAG axis was regulated} by selenite in vivo. We determined the in vivo effects of selenite on NB4 cells in a nude mouse model. This model has been built and demonstrated previously. Briefly, 5-weekold mice were injected with NB4 cells subcutaneously. After tumors were palpable, the mice were divided into two groups randomly. After selenite was given through intraperitoneal injection for $\sim 18$ days, the mice were killed for analysis. First, by hematoxylin-eosin staining ( $\mathrm{HE})$, we observed significant tumor cell death after selenite injection (Figure 5a). Second, through immunohistochemical staining, the differing levels of survivin-2B, IKK alpha, P73, UVRAG and the markers for apoptosis and autophagy were similar to those found in our in vitro results (Figure $5 b$ ). Finally, we

Figure 3 Survivin-2B accumulated and stabilized IKK alpha in the nucleus and regulated the switch from autophagy to apoptosis. (a) GST pull-down assay was used to determine that IKK alpha interacted with survivin-2B indeed. About $10^{6}$ cells were collected and lysed. The lysates were incubated with GST-survivin-2B and glutathione agarose beads. The interaction between IKK alpha and survivin-2B was determined by western blotting. (b) IKK alpha interacted with survivin-2B. After treatment with $20 \mu \mathrm{M}$ selenite for $24 \mathrm{~h}$, whole-cell lysates were extracted and immunoprecipitated with IKK alpha antibody. The interaction between IKK alpha and survivin-2B was determined by western blotting. (c) IKK alpha in the nucleus was decreased. After the cells were exposed to $20 \mu \mathrm{M}$ selenite for $12 \mathrm{~h}$, the nuclear and cytoplasmic fractions were extracted. The amount of IKK alpha in the nucleus was determined, whereas B23 and $\beta$-actin were used as loading controls for the nuclear and cytoplasmic fractions, respectively. (d) Survivin-2B colocalized with IKK alpha. After NB4 cells were exposed to selenite $(20 \mu \mathrm{M}), \mathrm{As}_{2} \mathrm{O}_{3}(0.8 \mu \mathrm{M}), \mathrm{H}_{2} \mathrm{O}_{2}(100 \mu \mathrm{M})$ and ATRA $(2 \mu \mathrm{M})$ for $24 \mathrm{~h}$, IKK alpha and survivin-2B were indirectly labeled with primary antibodies and either an FITC-conjugated or Cy3-conjugated secondary antibody, respectively. The images were visualized with a confocal microscope. The scale bar represents $10 \mu \mathrm{m}$. (e) Survivin-2B promoted autophagy. Cells were transfected with survivin-2B overexpression plasmids and LC3 was indirectly labeled. The images were visualized with a Zeiss microscope. The scale bar represents $10 \mu \mathrm{m}$. The degree of punctate aggregation was represented as LC3 dot-positive cells (with more than 10 dots)/100 cells. ${ }^{*} P<0.05$. (f) Survivin-2B prevented selenite inhibitory effects on autophagy. After cells were transfected with survivin-2B overexpression plasmids and selenite worked for another $24 \mathrm{~h}$, whole-cell lysates were extracted and alterations of Beclin-1 and LC3II were analyzed by western blotting. ( $\mathbf{g}-\mathbf{i})$ Selenite affected autophagy and apoptosis by regulating the levels of the survivin-2B protein. After being transfected (with siRNA targeting survivin-2B or survivin-2B overexpression plasmids) or pretreated with YM155 (500 nM) for $1 \mathrm{~h}$, NB4 cells were treated with $20 \mu \mathrm{M}$ selenite for $24 \mathrm{~h}$. The levels of C-caspase 9, C-caspase 3, C-PARP, UVRAG, IKK alpha and survivin-2B were detected by western blotting. (j) Survivin-2B regulated nuclear content of IKK alpha. After being transfected with survivin-2B overexpression plasmids, cells were treated with YM155 before selenite treated for another $24 \mathrm{~h}$. IKK alpha and survivin-2B were indirectly labeled with primary antibodies and either an FITC-conjugated or Cy3-conjugated secondary antibody, respectively. The images were visualized with a confocal microscope. The scale bar represents $20 \mu \mathrm{m}$. (k) Survivin-2B accumulated IKK alpha in the nucleus. After survivin-2B overexpression plasmids were transfected and selenite treated for another $12 \mathrm{~h}$, cytoplasmic and nuclear fractions were extracted. The contents of survivin-2B and IKK alpha at each fraction were analyzed by western blotting. (I) Survivin-2B also regulated IKK alpha and UVRAG in Jurkat and HCT116 cells. After cells were transfected with siRNA targeting survivin-2B for $12 \mathrm{~h}$, levels of IKK alpha, P73 and UVRAG were detected 
a

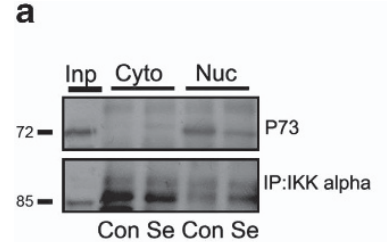

C
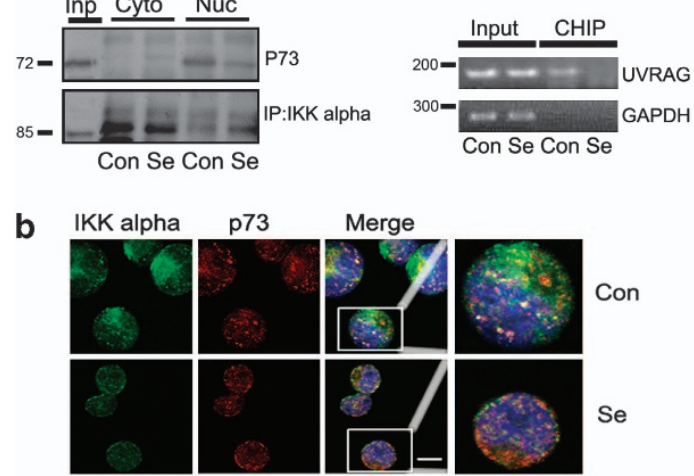

p73

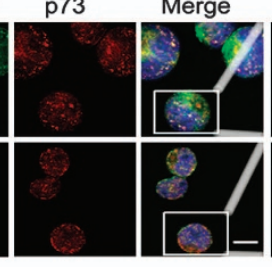

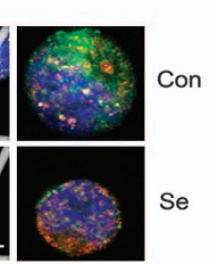

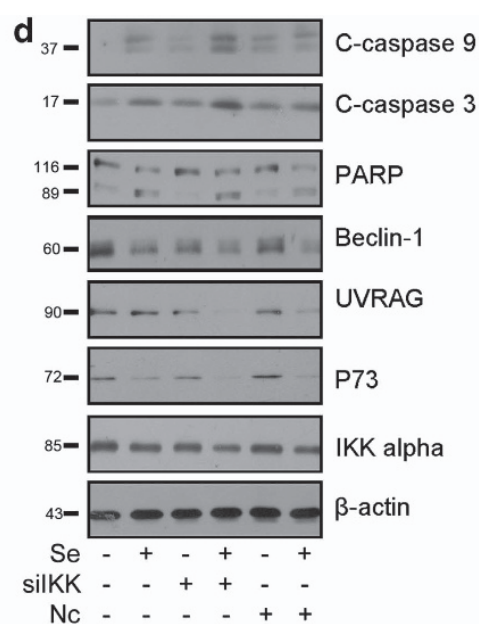

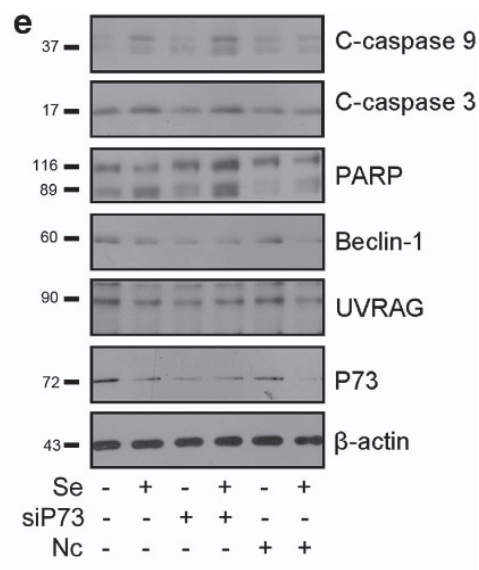

Figure 4 IKK/P73 participated in the regulation of UVRAG expression. (a) IKK alpha interacted with P73. After treatment with $20 \mu \mathrm{M}$ selenite for $12 \mathrm{~h}$, cytoplasm and nuclear fractions were extracted and immunoprecipitated with IKK alpha antibody. The interaction between IKK alpha and P73 was determined by western blotting. (b) P73 colocalized with IKK alpha. After exposure to $20 \mu \mathrm{M}$ selenite for $24 \mathrm{~h}$, IKK alpha and P73 were indirectly labeled with their own primary antibody and either an FITC-conjugated or Cy3-conjugated secondary antibody, respectively. The images were visualized with a confocal microscope. The scale bar represents $10 \mu \mathrm{m}$. (c) P73 directly bound to the promoter of UVRAG. After ChIP assay that was described previously was performed, PCR analysis was carried out. GAPDH was used as a negative control for ChIP sample and loading control for input sample. (d) IKK alpha regulated apoptosis and autophagy in NB4 cells. After being transfected with siRNA targeting IKK alpha and selenite treated for another $24 \mathrm{~h}$, levels of C-caspase 9, C-caspase 3, C-PARP, Beclin-1, UVRAG and P73 were detected. (e) P73 regulated apoptosis and autophagy in NB4 cells. After being transfected with siRNA targeting P73 and treatment with $20 \mu \mathrm{M}$ selenite for $24 \mathrm{~h}$, levels of C-caspase 9, C-caspase 3, C-PARP, Beclin-1 and UVRAG were detected

extracted whole-cell lysates from the tumor cells and assessed alterations in the levels of these proteins, which were consistent with our in vitro findings (Figure $5 \mathrm{c}$ ).

\section{Discussion}

Our previous study reported that Akt, ERK, P53 and Hsp90 participated in regulating the selenite-induced apoptosis and autophagy of leukemia cells. ${ }^{22}$ In the current study, we found that survivin-2B regulated apoptosis by mechanisms different from those of wild-type survivin that had been demonstrated previously. Moreover, the antiapoptotic role of survivin-2B was linked with the nuclear accumulation of IKK alpha. Such nuclear accumulation was necessary for the stability of P73 and the expression of UVRAG. Below, we discuss in detail the mechanisms by which survivin-2B regulates apoptosis and autophagy.

Autophagy, also known as type II programmed cell death, has been a popular area of investigation in recent years; however, the relationship between autophagy and apoptosis has remained unclear. In the past few years, some molecules, such as P53, mTOR, HMGB1 and HSP90, were identified as the crosstalk between apoptosis and autophagy. ${ }^{23}$ Some researchers indicated that autophagy was protective in some cancer cell lines, because it could clear damaged organelles, and several autophagy-related proteins, such as beclin-1, were reported to inhibit antiapoptotic protein degradation. ${ }^{24}$ However, others demonstrated that excessive autophagy could induce cell death. Recently, some researchers indicated that autophagosomes provide a platform for apoptosis induction. ${ }^{25}$ Therefore, to explore the relationship between autophagy and apoptosis is attractive and worthy. In the current study, we observed that selenite caused the protective autophagy to become apoptosis in NB4 cells. We concluded that the attenuation of protective autophagy was related to IAP proteins.
Survivin is known as an inhibitor of apoptosis protein and is usually overexpressed in the tumor tissue. As a result, analogs and inhibitors of survivin have potential antitumor activity. YM155 is a novel suppressor of survivin family members; in the recent years, it has been reported to regulate autophagy, but the exact mechanisms are unclear. It appeared to us that some survivin family members might regulate autophagy. Along with the in-depth study of survivin, several splice variants have been discovered and new functions are being explored gradually. Survivin-2B is one of the splice variants of survivin. Its function in regulating apoptosis is dependent on the cell type. However, whether survivin-2B regulates autophagy is still not known. In this report, we indicated that survivin-2B protected NB4 cells and promoted autophagy. We have also found that some researchers had proved that in AML patients survivin-2B might have protective effects. Therefore, our findings were supported in some way. In the present study, we also compared alterations of survivin and survivin-2B after different chemicals' exposure. We discovered that ATRA could not altered survivin similarly with IKK alpha, P73 and autophagy markers. It helped us identify the potential regulatory status of survivin-2B, whereas it did not mean that survivin would not have an important role in regulating NB4 cell death after selenite treatment. Moreover, the results in Figure $3 \mathrm{j}$ had indicated that inhibition of survivin and survivin-2B expression with YM155 could lead to the decrease of nuclear IKK alpha, whereas on that basis only expression of survivin-2B could rescue the decrease of nuclear content of IKK alpha. In some way, it indicated that although survivin might have effects on cell death determination, it worked independently of survivin-2B. The relationship between survivin and survivin-2B in selenite-treated NB4 cells would be discussed in our future work. 


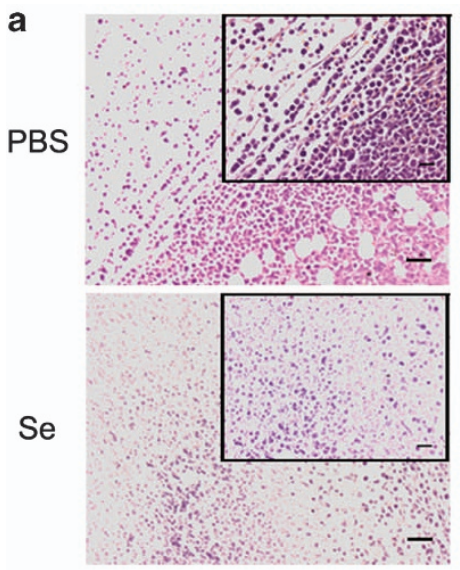

b

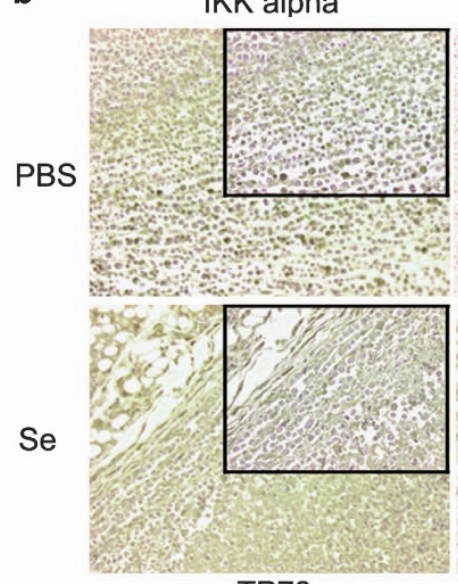
TP73

C

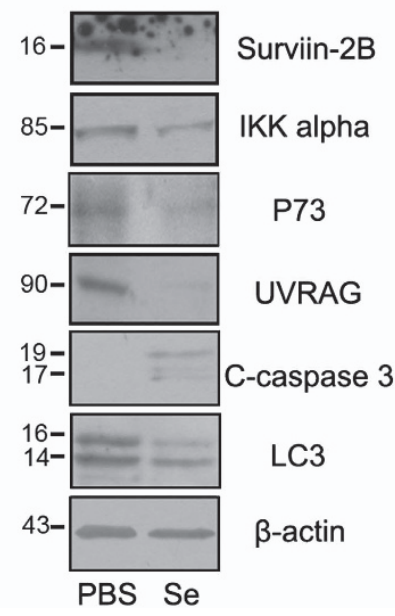

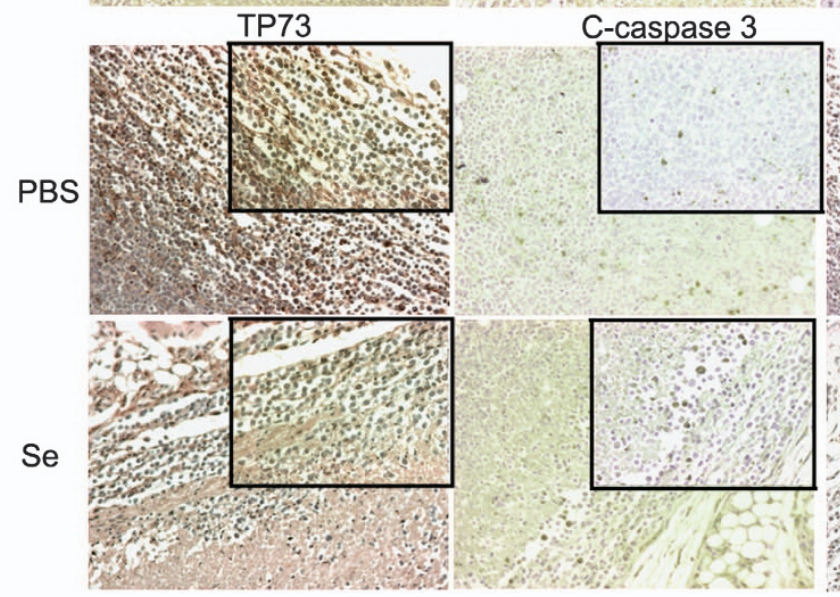

Survivin-2B
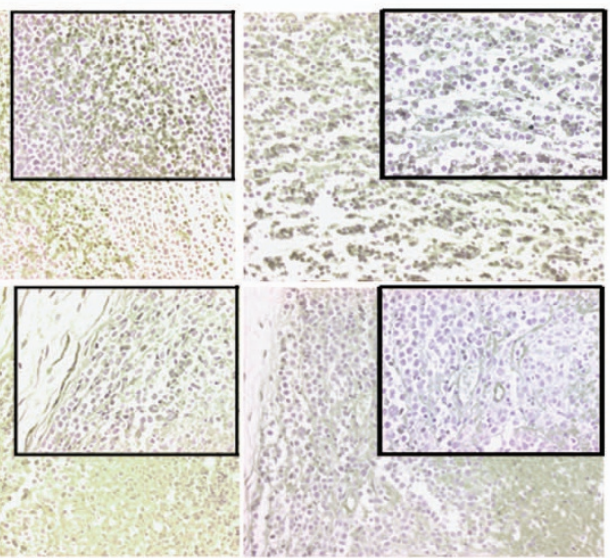

UVRAG

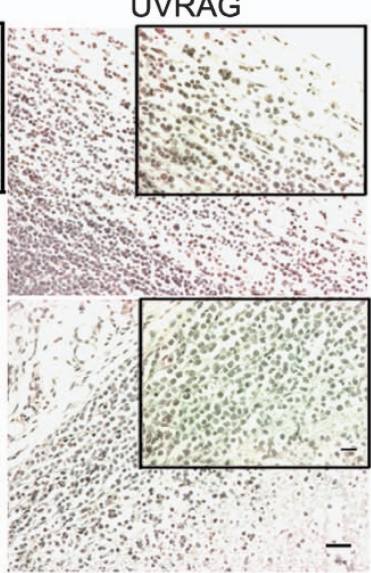

Figure 5 Survivin-2B and IKK alpha were regulated by selenite in vivo. (a) Selenite (3 mg/kg/day) induced the death of NB4 tumor cells in vivo. After HE staining, images were visualized with a Zeiss microscope. The scale bar in the larger image represents $50 \mu \mathrm{m}$, whereas that in the smaller image represents $25 \mu \mathrm{m}$. (b) Alterations induced by selenite $(3 \mathrm{mg} / \mathrm{kg} /$ day) in IKK alpha, survivin-2B, P73, UVAG, cleaved caspase 3 and LC3 were further confirmed by immunohistochemical staining. After immunohistochemical staining with antibodies, the images were visualized with a Zeiss microscope. The scale bar in the larger image represents $50 \mu \mathrm{m}$, whereas that in the smaller image represents $25 \mu \mathrm{m}$. (c) The levels of C-caspase 3, LC3, survivin-2B, IKK alpha, P73 and UVRAG altered similarly with our in vitro results. Whole tumor cell lysates were extracted, and the levels of C-caspase 3, LC3, IKK alpha, P73, UVRAG and survivin-2B were detected by western blotting

As we knew, survivin-2B could localize at the nucleus, microtubule organization center(MTOC) and cytoplasm. Survivin-2B localization at MTOC inhibited microtubule polymerization and therefore induced apoptosis. However, the function of survivin-2B localization at the nucleus has not been discussed. Here, we proved that survivin-2B accumulates IKK alpha in the nucleus. This result left us with two possibilities; one option is that survivin-2B controlled the localization of IKK alpha, and the other is that survivin-2B stabilized IKK alpha in the nucleus. We extracted cytoplasmic and nuclear fraction of selenite-treated NB4 cells after survivin-2B overexpression plasmids were transfected. We discovered that the seleniteinduced decrease of nuclear IKK alpha was inhibited along with enhanced expression of survivin-2B, whereas cytoplasmic IKK alpha was further downregulated. Therefore, we thought that survivin-2B might have an important role in regulating cellular localization of IKK alpha. In addition, from the western blot analysis in Figure 3, we could see that survivin-2B also worked on the regulation of IKK alpha protein level. Therefore, we speculated that survivin-2B must also regulate IKK alpha stability directly or indirectly (be dependent on the cytoplasmic localization of IKK alpha). Briefly, our previously published report had demonstrated that HSP90 interacted with IKK alpha and regulated its cytoplasmic function. As we knew, HSP90 regulated the activity and stability of some proteins. Its function in regulating IKK complex degradation had also been discovered. ${ }^{26}$ Therefore, we thought that survivin-2B must also regulate IKK alpha stability because of the partial selenite-induced inhibition of HSP90.

Next, we ask how the presence of IKK alpha in the nucleus regulates autophagy. Published articles indicated that cytoplasmic IKK alpha phosphorylated IKB and further activated NFKB. The activated NFKB could bind to the promoter of Beclin-1, which was an initiator of autophagy. Moreover, IKK alpha could also affect autophagy by regulating mTOR, JNK, AMPK and P53. ${ }^{27}$ In recent years, IKK alpha was shown to localize at the nucleus, and it either stabilized the transcription factor, $\mathrm{P} 73$, or promoted $\mathrm{NF} K \mathrm{~B}$ transcriptional activity by phosphorylating $\mathrm{H} 3$ at ser10. ${ }^{28-32}$ In this report, we discovered that IKK alpha stabilized P73 in the nucleus indeed, suggesting that survivin-2B regulated $\mathrm{P} 73$ by causing the 
accumulation of IKK alpha in the nucleus. P73 is a member of the P53 family and promotes the expression of tumor suppressor genes. ${ }^{33-35}$ Another question we addressed was how P73 affected autophagy, as studies have shown that P73 can regulate the expression of autophagy-related proteins such as UVRAG, Atg5 and Atg7. ${ }^{36-38}$ UVRAG interacts with PI3K class III and Beclin-1, which is necessary for the initiation of autophagy. ${ }^{39}$ In this study, we believed that $\mathrm{P} 73$ regulated UVRAG expression through direct binding to its promoter. Taken together, the survivin-2B-dependent nuclear accumulation of IKK alpha caused the transcription of UVRAG through the stabilization of P73. Finally, in a nude mouse model, we confirmed that the alterations of survivin-2B, IKK alpha, P73, UVRAG, LC3 and cleaved caspase 3 were similar to that observed in vitro. A decrease in nuclear IKK alpha was also observed in vivo, thereby validating our in vitro conclusions.

Survivin-2B is one of the splice variants of survivin. At present, the relationship between survivin-2B and cell death is not fully understood. Here, we explain that the antiapoptotic role of survivin-2B was related to protective autophagy induction. The pattern by which survivin-2B regulated autophagy was dependent on the content of nuclear IKK alpha, which stabilized P73 and further induced UVRAG expression (Figure 6). Understanding the antiapoptotic role of survivin-2B will be useful in improving cancer therapies. However, it is worth noting that autophagy in some cell lines has toxic effects, and P73 can also bind to the promoter of some proapoptotic family members; therefore, in that case, we cannot exclude the fact that survivin-2B has proapoptotic activity and all of these are necessary for researchers to explore. Finally, on the basis of the proapoptotic role of survivin-2B in other cell lines, some drugs that effectively induce apoptosis are most likely dependent on the expression of survivin-2B; in that case, combining these drugs with survivin inhibitors should be carefully done.

\section{Materials and Methods}

Chemicals. The bafilomycin A1 (B1793), 3MA (M9281) and sodium selenite (S-5261) were obtained from Sigma (St. Louis, MO, USA). The antibodies used for western blotting, including anti-IKK alpha (\#2682S), anti-Beclin-1 (\#3496P), antiLC3B (\#3868P), anti-caspase 3 (\#9661S) and anti-PARP (\#9546S), were obtained from Cell Signaling Technology (Danvers, MA, USA), whereas anti-P73 antibodies were purchased from Abgent (San Diego, CA, USA), and the anti-UVRAG (sc-82115) and anti-B23 (sc-32256) antibodies for western blots were purchased from Santa Cruz (Santa Cruz, CA, USA). For indirect immunofluorescence, antiIKK alpha (ab105923) antibodies were obtained from Abcam (Cambridge, UK), whereas anti-LC3 antibodies (M152-3) were purchased from MBL (Woburn, MA, USA) and anti-P73 antibodies (bs-1346R) were purchased from BIOSS (Beijing, China). For immunohistochemical staining, anti-IKK alpha (sc-7183) antibodies were purchased from Santa Cruz. Anti-survivin-2B (ab3729) was purchased from Abcam. The HRP-conjugated anti-goat (ZB-2306), anti-mouse (ZB-2305) and antirabbit (ZB-2301) antibodies were obtained from ZSGB-BIO (Beijing, China). The Cy3-conjugated anti-rabbit (89856) and FITC-conjugated anti-mouse (89750) antibodies were purchased from Jackson (West Grove, PA, USA).

Cell lines. NB4 and Jurkat cells were grown in RPMI 1640 medium containing 100 units/ml streptomycin, 100 units/ml penicillin and $10 \%$ advanced fetal bovine serum. HCT116 cells were grown in DMEM medium. The cells were incubated in $5 \% \mathrm{CO}_{2}$ at $37^{\circ} \mathrm{C}$.

Western blotting. Approximately $10^{6}$ cells were collected after they were exposed to selenite or other treatments. The cells were washed twice with ice-cold PBS, resuspended in RIPA buffer (1 mM EDTA, $1 \mathrm{mM}$ EGTA, $20 \mathrm{nM}$ Tris, pH 7.5, $1 \%$ Triton X-100, $150 \mathrm{mM} \mathrm{NaCl}, 1 \mathrm{mM} \beta$-glycerolphosphate, $2.5 \mathrm{mM}$ sodium pyrophosphate, $1 \mathrm{mM} \mathrm{Na}_{3} \mathrm{VO}_{4}, 1 \mu \mathrm{g} / \mathrm{mL}$ leupeptin and $1 \mathrm{mM}$ phenylmethanesulfonyl fluoride) and ultrasonicated at $4{ }^{\circ} \mathrm{C}$. After centrifugation for $15 \mathrm{~min}$ at $12000 \times \mathrm{g}$ and $4^{\circ} \mathrm{C}$, the proteins were separated by SDS-PAGE and then transferred to a nitrocellulose membrane. After being blocked with $5 \%$ fat-free milk, the membranes were incubated with primary antibodies at $4{ }^{\circ} \mathrm{C}$ overnight. The membranes were washed three times with TBST and then incubated with the secondary antibodies at room temperature for $\sim 1-2 \mathrm{~h}$. Finally, the membranes were washed an additional three times and probed.

Indirect immunofluorescence. Approximately $10^{6}$ cells were collected and washed twice with ice-cold PBS. The cells were transferred to slides and fixed with ice-cold methanol for $10 \mathrm{~min}$, and then washed three times. Triton X-100 $(0.1 \%)$ was added, and the cells were blocked with $2-5 \%$ BSA and incubated with primary antibodies overnight at $4{ }^{\circ} \mathrm{C}$. After a second round of washing, the cells were incubated with FITC-conjugated or Cy3-conjugated secondary antibodies for $1 \mathrm{~h}$ at room temperature. Last, the images were visualized with a confocal microscope.

Immunoprecipitation and GST pull-down. Cells $\left(1 \times 10^{7}\right)$ were collected and washed twice with ice-cold PBS. The pellets were resuspended in RIPA buffer and placed in an ice bath for an additional $30 \mathrm{~min}$. The samples were centrifuged at $12000 \times \mathrm{g}$ and $4^{\circ} \mathrm{C}$ for $10 \mathrm{~min}$, and the supernatant was collected. An equal amount of supernatant from each treatment condition was incubated with antibodies overnight at $4^{\circ} \mathrm{C}$. The remainder of each sample was used as the input sample. After adding protein $A+G$ beads and incubating for an additional $3 \mathrm{~h}$ at $4^{\circ} \mathrm{C}$, each sample was washed with RIPA lysis buffer three times. Finally, the mixture of beads and SDS loading buffer was boiled for $10 \mathrm{~min}$, and the supernatant was collected. For pull-down assay, after cells were lysed, GSTsurvivin- $2 \mathrm{~B}$ and glutathione agarose beads were added and the mixture was rotated for $4 \mathrm{~h}$ at $4{ }^{\circ} \mathrm{C}$. After washing with RIPA buffer three times, the GSTsurvivin-2B was eluted and separated with SDS-PAGE.

Detection of apoptotic ratio. Approximately $10^{5}$ cells were collected and washed twice with ice-cold PBS. Each sample was treated with a mixture of Annexin $\mathrm{V}$ and PI for $15 \mathrm{~min}$ in the dark. The cell fragments were filtered out, and

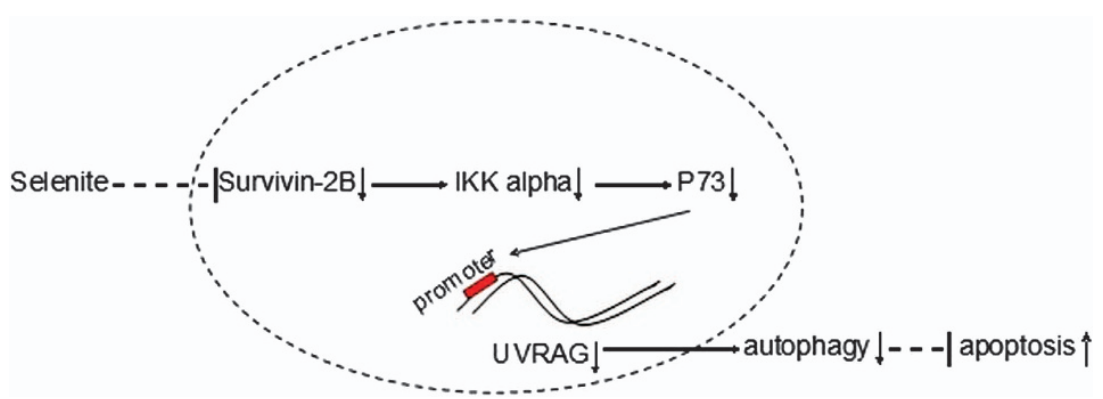

Figure 6 Survivin-2B accumulated IKK alpha in the nucleus, which further stabilized P73, a transcription factor of UVRAG. P73 in the nucleus upregulated UVRAG, an initiator of autophagy, and therefore regulated protective autophagy in NB4 cells 
the apoptotic ratio was detected by the Accuri C6 flow cytometry (Accuri Cytometers Inc., Ann Arbor, MI, USA).

Transfections. SiRNAs targeting Beclin-1 (5'-GAUUGAAGACACAGGAGG CTT-3'), LC3B (5'-GCTTACAGCTCAATGCTAA-3'), survivin-2B (5'-GATCACGAG AGAGGAACAT-3'), IKK alpha (5'-GCCTTACACAGCCACTGTT-3'), P73 (5'-CCC AAGGGT TACAGAGCAT- $3^{\prime}$ ) and the corresponding negative controls for each siRNA were synthesized by Genepharma (Shanghai, China). The cells $\left(10^{6}\right)$ were collected, washed twice with Opti-MEM medium and then transfected with a mixture of $200 \mathrm{nM}$ siRNA or $10 \mu \mathrm{g}$ plasmids and lipofectamine 2000 (Invitrogen, Carlsbad, CA, USA) in Opti-MEM (Gibco, Grand Island, NY, USA) for $\sim 6 \mathrm{~h}$. After transfection, the cells were cultured for $12 \mathrm{~h}$ and then treated with sodium selenite for an additional $24 \mathrm{~h}$.

Plasmids. The cDNA of survivin-2B from Jurkat cells was amplified by PCR. The PCR product and the PCDNA 6 plasmid were double digested with the $B a m H I / E c o R I$ enzymes. The double-digestion product was ligated using the T4 ligase. After transformation into $\mathrm{DH} 5 \alpha$ cells, the clone that yielded a positive result was chosen for further DNA sequencing. Finally, the plasmids were extracted for use.

Tumor xenograft. This model has been built and demonstrated previously. ${ }^{40}$ Briefly, NB4 leukemia cells were administered subcutaneously to the nude mice. After the tumors were palpable, the mice were divided into two groups, and selenite $(3 \mathrm{mg} / \mathrm{kg} /$ day and injection every two days) was dissolved in PBS and injected intraperitoneally. The control group was injected with PBS alone. After 18 days, the mice were killed for analysis. The Helsinki Declaration of 1975 was followed.

Immunohistochemical staining. First, the fixed tissues were wrapped in paraffin. After the tissues were sectioned into slices, they were transferred onto slides. The slides then underwent dewaxing and hydration. Before incubation with primary antibodies overnight at $4{ }^{\circ} \mathrm{C}$, the tissues were blocked with $3 \%$ peroxide in methanol. After a second round of washing, the tissues were incubated with the secondary antibodies for $3 \mathrm{~h}$ at room temperature and then were treated with $D A B$ and labeled with Mayer's hematoxylin. The slides underwent dehydration in increasing concentrations of ethanol and were then clarified with xylene. Finally, the slides were mounted using mounting medium.

HE staining. After undergoing hydration as described above, the tumor samples were labeled with the Harris hematoxylin and washed with running water for $\sim 2 \mathrm{~min}$. The slides were then placed in $1 \%$ hydrochloric acid in $75 \%$ ethanol for $30 \mathrm{~s}$ and labeled with eosin for $10 \mathrm{~min}$. Finally, the slides were clarified with xylene and mounted using mounting medium.

Extraction of nuclear fractions. To extract the nuclear fractions, cells $\left(10^{6}\right)$ were collected and washed twice with ice-cold PBS. The pellets were resuspended in cytoplasmic extraction buffer immediately and then lysed on ice for $\sim 30 \mathrm{~min}$. After lysis, the mixture was centrifuged at $12000 \times \mathrm{g}$ and $4{ }^{\circ} \mathrm{C}$ for $10 \mathrm{~min}$, and the pellets were again resuspended in nuclear extraction buffer for a second round of lysis on ice. Each sample was again centrifuged at $12000 \times \mathrm{g}$ and $4{ }^{\circ} \mathrm{C}$ for $10 \mathrm{~min}$, and the supernatant was collected. Finally, the cytoplasmic and nuclear fractions were separated by SDS-PAGE for further analysis.

Electron microscopy. Cells of each treatment were collected and treated with $2.5 \%$ glutaraldehyde. The exact process had been demonstrated previously. ${ }^{7}$ The images were visualized by transmission electron microscopy.

Chromatin immunoprecipitation. Simple ChIP Enzymatic Chromatin IP Kit (Cell Signaling Technology) was used. About $10^{6}$ cells were collected and they underwent the cross-link process with formaldehyde for about $10 \mathrm{~min}$ at room temperature. The cross-link process was stopped with glycine at a final concentration at $0.125 \mathrm{M}$. After washing with ice-cold PBS three times, the pellets were resuspended with buffer $A$ on ice for $10 \mathrm{~min}$. Then the nucleus section was collected and incubated with buffer B. The product was then digested with micrococcal nuclease, which finally stopped with 0.5 M EDTA on ice. After centrifugation at a speed of 13000 r.p.m. for about $10 \mathrm{~min}$ at $4{ }^{\circ} \mathrm{C}$, the pellets were resuspended and put on ice for about $10 \mathrm{~min}$. After incubation with P73 antibody for about $12 \mathrm{~h}$ at $4{ }^{\circ} \mathrm{C}$, the mixture was added with ChIP-Grade Protein G Agarose
Beads rotating for another $3 \mathrm{~h}$ at $4{ }^{\circ} \mathrm{C}$. After washing, DNA was eluted for subsequent PCR analysis. The primers(sense: $5^{\prime}$-CATCCTGCGTGGTGGCTCT-3'; anti-sense: $5^{\prime}$-CTCACTGGTTGCCGTGGT-3') for P73 site at the promoter of UVRAG were purchased from Sangon Biotech.

RT-PCR. Trizol agent (Invitrogen) was used to extract RNA. This process was carried out with M-MLV reverse transcriptase, dNTPs and oligo dT primer that were provided by Promega (Madison, WI, USA). Primers for LC3 (forward: $5^{\prime}-A$ TGCCGTCGGAGAAGACC-3'; reverse: 5'-TTACACTGACAATTTCATCCCGAA C-3') and GAPDH (forward: 5'-ATGGTTTACATGTTCCAATATGATTCCAC-3'; reverse: $5^{\prime}$-TTACTCCTTGGAGGCCATGT-3') were purchased from Sangon Biotech (Shanghai, China).

Statistical analysis. All statistical analyses were performed using a two-tailed Student's $t$-test, and $P<0.05$ was considered the threshold of significance. Other experiments were also repeated at least three times.

\section{Conflict of Interest}

The authors declare no conflict of interest.

Acknowledgements. The work was supported by the National Natural Science Foundation of China (No. 31170788 and No. 31340037), the National Natural Science Foundation for Young Scholars of China (No. 31101018), the State Key Laboratory Special Fund (No. 2060204), the Natural Science Foundation of Beijing (No. 5082015) and the Ministry of Education of China's Doctor Training Unit (No. 20091106110025)

1. Wu WK, Coffelt SB, Cho CH, Wang XJ, Lee CW, Chan FK et al. The autophagic paradox in cancer therapy. Oncogene 2012; 31: 939-953.

2. Guan L, Han B, Li J, Li Z, Huang F, Yang Y et al. Exposure of human leukemia NB4 cells to increasing concentrations of selenite switches the signaling from pro-survival to pro-apoptosis. Ann Hematol 2009; 88: 733-742.

3. Brozmanova J, Manikova D, VIckova V, Chovanec M. Selenium: a double-edged sword for defense and offence in cancer. Arch Toxicol 2010; 84: 919-938.

4. Guan L, Han B, Li Z, Hua F, Huang F, Wei W et al. Sodium selenite induces apoptosis by ROS-mediated endoplasmic reticulum stress and mitochondrial dysfunction in human acute promyelocytic leukemia NB4 cells. Apoptosis 2009; 14: 218-225.

5. Han B, Wei W, Hua F, Cao T, Dong H, Yang T et al. Requirement for ERK activity in sodium selenite-induced apoptosis of acute promyelocytic leukemia-derived NB4 cells. J Biochem Mol Biol 2007; 40: 196-204.

6. Shi K, Jiang Q, Li Z, Shan L, Li F, An J et al. Sodium selenite alters microtubule assembly and induces apoptosis in vitro and in vivo. J Hematol Oncol 2013; 6: 7.

7. Ren $Y$, Huang F, Liu Y, Yang Y, Jiang Q, Xu C. Autophagy inhibition through PI3K/Akt increases apoptosis by sodium selenite in NB4 cells. BMB Rep 2009; 42: 599-604.

8. An JJ, Shi KJ, Wei W, Hua FY, Ci YL, Jiang $Q$ et al. The ROS/JNK/ATF2 pathway mediates selenite-induced leukemia NB4 cell cycle arrest and apoptosis in vitro and in vivo. Cell Death Dis 2013; 4: e973.

9. Jiang Q, Wang Y, Li T, Shi K, Li Z, Ma Y et al. Heat shock protein 90-mediated inactivation of nuclear factor-kappaB switches autophagy to apoptosis through becn1 transcriptional inhibition in selenite-induced NB4 cells. Mol Biol Cell 2011; 22: 1167-1180.

10. Kim EH, Sohn S, Kwon HJ, Kim SU, Kim MJ, Lee SJ et al. Sodium selenite induces superoxide-mediated mitochondrial damage and subsequent autophagic cell death in malignant glioma cells. Cancer Res 2007; 67: 6314-6324.

11. Kralova V, Benesova S, Cervinka M, Rudolf E. Selenite-induced apoptosis and autophagy in colon cancer cells. Toxicol In Vitro 2012; 26: 258-268.

12. Jiang $Q$, Li F, Shi K, Yang Y, Xu C. Sodium selenite-induced activation of DAPK promotes autophagy in human leukemia HL60 cells. BMB Rep 2012; 45: 194-199.

13. Park SH, Kim JH, Chi GY, Kim GY, Chang YC, Moon SK et al. Induction of apoptosis and autophagy by sodium selenite in A549 human lung carcinoma cells through generation of reactive oxygen species. Toxicol Lett 2012; 212: 252-261.

14. Roca H, Varsos ZS, Mizutani K, Pienta KJ. CCL2, survivin and autophagy: new links with implications in human cancer. Autophagy 2008; 4: 969-971.

15. Niu TK, Cheng Y, Ren X, Yang JM. Interaction of Beclin 1 with survivin regulates sensitivity of human glioma cells to TRAIL-induced apoptosis. FEBS Lett 2010; 584: 3519-3524.

16. Wang Q, Chen Z, Diao X, Huang S. Induction of autophagy-dependent apoptosis by the survivin suppressant YM155 in prostate cancer cells. Cancer Lett 2011; 302: 29-36.

17. Chiu HW, Ho YS, Wang YJ. Arsenic trioxide induces autophagy and apoptosis in human glioma cells in vitro and in vivo through downregulation of survivin. J Mol Med (Berl) 2011; 89: 927-941. 
18. Ling X, Cheng Q, Black JD, Li F. Forced expression of survivin-2B abrogates mitotic cells and induces mitochondria-dependent apoptosis by blockade of tubulin polymerization and modulation of Bcl-2, Bax, and survivin. J Biol Chem 2007; 282: 27204-27214.

19. Mahotka C, Wenzel M, Springer E, Gabbert HE, Gerharz CD. Survivin-deltaEx3 and survivin-2B: two novel splice variants of the apoptosis inhibitor survivin with different antiapoptotic properties. Cancer Res 1999; 59: 6097-6102.

20. Wagner M, Schmelz K, Wuchter C, Ludwig WD, Dorken B, Tamm I. In vivo expression of survivin and its splice variant survivin-2B: impact on clinical outcome in acute myeloid leukemia. Int J Cancer 2006; 119: 1291-1297.

21. Zhu N, Gu L, Findley HW, Li F, Zhou M. An alternatively spliced survivin variant is positively regulated by p53 and sensitizes leukemia cells to chemotherapy. Oncogene 2004; 23 : 7545-7551.

22. Li Z, Shi K, Guan L, Cao T, Jiang Q, Yang $Y$ et al. ROS leads to MnSOD upregulation through ERK2 translocation and p53 activation in selenite-induced apoptosis of NB4 cells. FEBS Lett 2010; 584: 2291-2297.

23. Livesey KM, Kang R, Vernon P, Buchser W, Loughran P, Watkins SC et al. p53/HMGB1 complexes regulate autophagy and apoptosis. Cancer Res 2012; 72: 1996-2005.

24. Unuma K, Aki T, Matsuda S, Funakoshi T, Yoshida KI, Uemura K. Inducer of heme oxygenase-1 cobalt protoporphyrin accelerates autophagy and suppresses oxidative damages during lipopolysaccharide treatment in rat liver. Hepatol Res 2012; 43: 91-96.

25. Young MM, Takahashi Y, Khan O, Park S, Hori T, Yun J et al. Autophagosomal membrane serves as platform for intracellular death-inducing signaling complex (iDISC)-mediated caspase-8 activation and apoptosis. J Biol Chem 2012; 287: 12455-12468.

26. Lee $\mathrm{KH}$, Jang $\mathrm{Y}$, Chung $\mathrm{JH}$. Heat shock protein 90 regulates IkappaB kinase complex and NF-kappaB activation in angiotensin II-induced cardiac cell hypertrophy. Exp $\mathrm{Mol}$ Med 2010; 42: 703-711

27. Criollo A, Senovilla L, Authier H, Maiuri MC, Morselli E, Vitale I et al. IKK connects autophagy to major stress pathways. Autophagy 2010; 6: 189-191.

28. Yoshida K, Ozaki T, Furuya K, Nakanishi M, Kikuchi H, Yamamoto H et al. ATM-dependent nuclear accumulation of IKK-alpha plays an important role in the regulation of p73-mediated apoptosis in response to cisplatin. Oncogene 2008; 27: 1183-1188.

29. Furuya K, Ozaki T, Hanamoto T, Hosoda M, Hayashi S, Barker PA et al. Stabilization of p73 by nuclear lkappaB kinase-alpha mediates cisplatin-induced apoptosis. J Biol Chem 2007; 282: 18365-18378.
30. Ozaki T, Nakagawara A. P53-independent apoptosis through a novel ATM/IKK-alpha/p73mediated apoptotic pathway. Seikagaku 2008; 80: 409-413.

31. Yamamoto Y, Verma UN, Prajapati S, Kwak YT, Gaynor RB. Histone H3 phosphorylation by IKK-alpha is critical for cytokine-induced gene expression. Nature 2003; 423: 655-659.

32. Anest V, Hanson JL, Cogswell PC, Steinbrecher KA, Strahl BD, Baldwin AS. A nucleosomal function for lkappaB kinase-alpha in NF-kappaB-dependent gene expression. Nature 2003; 423: 659-663.

33. Okazaki T, Kageji T, Kuwayama K, Kitazato KT, Mure H, Hara K et al. Up-regulation of endogenous PML induced by a combination of interferon-beta and temozolomide enhances p73/YAP-mediated apoptosis in glioblastoma. Cancer Lett 2012; 323: 199-207.

34. Al-Bahlani S, Fraser M, Wong AY, Sayan BS, Bergeron R, Melino G et al. P73 regulates cisplatin-induced apoptosis in ovarian cancer cells via a calcium/calpain-dependent mechanism. Oncogene 2011; 30: 4219-4230.

35. Stiewe T, Putzer BM. p73 in apoptosis. Apoptosis 2001; 6: 447-452.

36. Crighton D, O'Prey J, Bell HS, Ryan KM. p73 regulates DRAM-independent autophagy that does not contribute to programmed cell death. Cell Death Differ 2007; 14: 1071-1079.

37. Rosenbluth JM, Pietenpol JA. mTOR regulates autophagy-associated genes downstream of p73. Autophagy 2009; 5: 114-116.

38. Humbert M, Mueller C, Fey MF, Tschan MP. Inhibition of damage-regulated autophagy modulator-1 (DRAM-1) impairs neutrophil differentiation of NB4 APL cells. Leuk Res 2012; 36: $1552-1556$

39. Kang R, Zeh HJ, Lotze MT, Tang D. The Beclin 1 network regulates autophagy and apoptosis. Cell Death Differ 2011; 18: 571-580.

40. Li F, Jiang Q, Shi KJ, Luo H, Yang Y, Xu CM. RhoA modulates functional and physical interaction between ROCK1 and Erk1/2 in selenite-induced apoptosis of leukaemia cells. Cell Death Dis 2013; 4: e708.

(c) () (2) Cell Death and Disease is an open-access journa published by Nature Publishing Group. This work is licensed under a Creative Commons Attribution-NonCommercialShareAlike 3.0 Unported License. To view a copy of this license, visit http://creativecommons.org/licenses/by-nc-sa/3.0/

Supplementary Information accompanies this paper on Cell Death and Disease website (http://www.nature.com/cddis) 